\title{
ULK1 forms distinct oligomeric states and nanoscopic morphologies during autophagy initiation
}

Chiranjib Banerjee ${ }^{1}$, Dushyant Mehra ${ }^{1,3}$, Daihyun Song ${ }^{2}$, Angel Mancebo ${ }^{1}$, Do-Hyung Kim,** and Elias M. Puchner ${ }^{1, *}$

1. School of Physics and Astronomy, University of Minnesota, Twin Cities

2. Department of Biochemistry, Molecular Biology, and Biophysics, University of Minnesota, Twin Cities

3. Department of Biomedical Engineering and Physiology, Mayo Clinic, Rochester, MN

*Corresponding authors: epuchner@umn.edu and dhkim@umn.edu 


\begin{abstract}
Autophagy is an evolutionarily conserved process for the degradation and recycling of intracellular components. Although autophagy has been extensively studied, it still remains unclear how autophagosome formation occurs in response to starvation. Here we combined CRISPR-cas9assisted genome-editing with quantitative Photoactivated Localization Microscopy (qPALM) to analyze the nanoscopic spatial distribution and oligomeric states of endogenous ULK1, the central autophagy induction regulator with single molecule sensitivity. Amino acid starvation induced a small fraction of ULK1 molecules to localize to arc-shaped and spherical structures with radii up to $300 \mathrm{~nm}$ and with more than 30 ULK1 molecules. These starvation-induced structures with high ULK1 content occurred only when ULK1 was colocalized with Atg13 and within $100 \mathrm{~nm}$ distance to the endoplasmic reticulum. This analysis revealed that a threshold number of ULK1 molecules around 30 is necessary to drive the formation of early autophagic ULK1 structures under starvation, providing an unprecedented quantitative insight into a hierarchical transition of ULK1 states during autophagy initiation.
\end{abstract}

Keywords: Autophagy, ULK1, Atg13, PALM/STORM, single molecule sensitivity, photoswitchable fluorescent protein, cross correlation, CRISPR/Cas9, endogenous tagging. 


\section{Introduction}

Macroautophagy (hereafter referred to as autophagy) is an evolutionarily conserved process in eukaryotic cells that degrades intracellular organelles and macromolecules in the lysosome. Autophagy plays a fundamental role in maintaining the balance between production, degradation, and recycling of cellular constituents. Dys-regulation of autophagy is closely related to various diseases, such as cancer, neurodegeneration, and diabetes ${ }^{1,2}$. Autophagy is mainly induced under starvation of amino acids or other stress conditions via suppression of mechanistic target of rapamycin complex 1 (mTORC1), the central nutrient-sensitive protein kinase complex ${ }^{3-5}$. Despite the recent progress in our understanding of autophagy, the exact mechanism of autophagy initiation remains unclear.

Autophagy initiates with the formation of the autophagosome, the double membrane structure that encompasses its cargo. According to the prevalent model, autophagosome formation starts at the endoplasmic reticulum (ER) exit sites or the ER-mitochondria contact sites with the formation of the phagophore, the precursor membrane structure for the autophagosome ${ }^{6-8}$. The phagophore membrane has also been shown to originate from late endosomes or lysosomes, the Golgi complex as well as mitochondria ${ }^{6-8}$. The key step for autophagosome formation is activation of the UNC51-like kinase 1 (ULK1) ${ }^{9-12}$. ULK1 forms a large protein complex by interacting with Atg13, FIP200 (FAK family kinase interacting protein $200 \mathrm{kDa}$ ), and Atg1019-16. ULK1, largely localizes on the ER or Atg9 compartments ${ }^{17}$ and forms puncta in the cytoplasm of starved cells prior to the formation of the phagophore ${ }^{18-20}$ This suggests that understanding how ULK1 puncta structures form is critical to clarify the mechanism of phagophore formation.

Prior studies employing confocal or other conventional fluorescence microscopy techniques have provided useful but limited knowledge on the nature of ULK1 puncta. Highly sensitive molecular imaging techniques have recently been developed, enabling to resolve intracellular structures below the optical diffraction limit. In particular, quantitative Photoactivated Localization Microscopy (qPALM) ${ }^{21,22}$ using irreversibly bleaching photoconvertible or photoswitchable fluorescent proteins (PSFPs) has enabled us to count the number of molecules in diffraction limited structures inside cells ${ }^{23-29}$. Analysis techniques have also been developed for PALM to determine the precise spatial distribution of signaling proteins ${ }^{30,31}$. 
Most fluorescence and super-resolution microscopy studies conducted with mammalian cells have relied on overexpression of fluorescently tagged proteins ${ }^{32}$. However, overexpression can cause artifacts for determining the oligomeric state and spatial distribution of proteins ${ }^{33}$. In addition, overexpression can abnormally alter the biological function of proteins ${ }^{34}$. A few recent studies have applied PALM with endogenously tagged proteins ${ }^{35-38}$. However, none of the studies could successfully quantify the number of proteins due to the challenges associated with the blinking behavior of PSFPs.

In this study, we have employed the CRISPR/Cas9-based genome-editing technique ${ }^{39}$ to introduce mEos2, one of the best characterized PSFPs ${ }^{26,40}$, into the ULK1 gene allele of HeLa cell genome. This allowed us to express endogenous ULK1 as a fusion with mEos2. By applying qPALM to the genome-modified cells, we have determined the nanoscopic distribution and the oligomeric state of endogenous ULK1 with single-molecule sensitivity. We were able to quantify a threshold number of ULK1 molecules that might reflect the state of ULK1 participating in autophagy initiation, providing an unprecedented level of insight into ULK1 multimerization and autophagy initiation.

\section{Results}

\section{Quantitative analysis of endogenous ULK1 clusters reveals its high-order multimeric states}

ULK1 forms puncta in the cytoplasm prior to autophagosome formation ${ }^{17,18,20}$. To characterize the nature of ULK1 puncta, we employed qPALM and quantified the multimeric states of ULK1 molecules. To avoid potential artifacts of qPALM analysis caused by overexpression, we engineered the HeLa cell genome with the CRISPER/Cas9-assisted genome editing technique ${ }^{39}$ introducing mEos 2 into the allele of the ULK1 gene (Fig. 1a). We selected genome-edited cells in which all endogenous ULK1 molecules were expressed as a fusion with mEos2 (Fig. 1b). The endogenously tagged ULK1 was confirmed to form functional complexes with Atg13 and FIP200 as similarly as untagged endogenous ULK1 (Fig. 1b), and it responded to amino acid starvation for its activation toward phosphorylation of Atg14 Ser29, an ULK1 target $\operatorname{site}^{20}$ (Fig. 1c). The genome-edited cells showed induction of Atg13 and LC3B puncta to a similar extent as 
unmodified HeLa cells under amino acid starvation (Supplementary Fig. 1). These results demonstrate that the functional properties of ULK1 have not been disturbed by genome editing.

Using the genome-edited cells, we analyzed PALM data of mEos2-ULK1 with the aim to determine the oligomeric states of ULK1 (Fig. 1d). Each time a single mEos2 molecule was photoactivated and fluoresced, its center of mass was determined by a Gaussian fit to its intensity profile. Since mEos2 like other fluorophores can emit a variable number of fluorescent burst that are separated by a variable short dark-time, a single molecule can appear as a cloud of localizations in raw PALM data (Fig. 1e, left) ${ }^{23}$. To correct for these clustering artifacts, we employed our previously developed method of grouping localizations originating from the same mEos 2 molecule to a single, photon weighted averaged position (Fig. 1e, right) ${ }^{26}$. This grouping requires two parameters: the maximum spatial spread of localizations from single mEos 2 molecules, and the maximum dark-time between them. The maximum spatial spread was determined with the radial distribution (or pair-correlation) analysis ${ }^{26,28}$, which quantifies the density of any pair of localizations as a function of their distance (Supplementary Fig. 2). The peak in the radial distribution function up to a distance of $80 \mathrm{~nm}$ represents the localization uncertainty in raw PALM images $^{26}$ and was used as the maximum spatial spread for grouping localization. Likewise, the distributions of all dark-times between fluorescent bursts from single mEos 2 molecules was used to determine the maximum time between fluorescent bursts for grouping (Supplementary Fig. 2e and Materials and Methods). By applying the dark-time parameter and the spatial spread of localizations, we corrected the PALM data to obtain the actual number of ULK1 molecules in ULK1 clusters with $\sim 20 \mathrm{~nm}$ resolution as well as the overall distribution of oligomeric states within a cell (Fig. 1d and e).

\section{Transiently overexpressed ULK1 forms different oligomerization patterns compared to endogenous ULK1.}

Super-resolution and conventional fluorescence imaging studies in mammalian cells have been mostly conducted using overexpressed fluorescently tagged proteins. Overexpression can disturb the stoichiometry of protein compositions in complexes or pathways. Both the sizes and intensities of ULK1 puncta were more heterogeneous and larger with overexpressed ULK1 compared to endogenous ULK1 (Fig. 2a). By applying qPALM analysis, we found that overexpressed ULK1 exhibits a different pattern of multimerization compared to endogenous ULK1 (Fig. 2b and c). In 
this analysis, all ULK1 molecules that appear within a distance of $400 \mathrm{~nm}$ from each other are considered to be in one structure or cluster (see also Materials and Methods). This distance was chosen for consistency since starvation-induced large ULK1 structures were later analyzed with this distance parameter. Endogenous ULK1 formed clusters containing a significantly lower number of ULK1 molecules compared to overexpressed ULK1. Using pair-correlation analysis, which quantifies the distribution of distances between all pairs of localizations ${ }^{30}$, we determined that the average distance of ULK1 molecules in clusters and their density were significantly larger with overexpressed ULK1 (Fig. 2c). These results suggest that overexpressed ULK1 behaves differently from endogenous ULK1 in forming the oligomeric states.

\section{Amino acid starvation induces large high-order ULK1 oligomers}

Having established qPALM with endogenously tagged ULK1, we asked how amino acid starvation affects the oligomeric states of ULK1. Regardless of amino acid presence or absence, mEos2ULK1 was distributed throughout the whole cytoplasm (Fig. 3a and b). Again, we performed qPALM analysis and grouped molecules appearing within a size threshold of $400 \mathrm{~nm}$, which was found to include starvation-induced large structures (Materials and Methods). Over 93\% of ULK1 molecules existed as clusters containing 1 to 10 molecules in either fed or starved conditions (Fig. 3c inset). Distinctly in starved cells, we started to observe emerging arc-shaped and spherical structures of various sizes (Fig. 3b). These structures could not be resolved in conventional fluorescence images. Analysis of the oligomeric states revealed that the largest number of ULK1 molecules in one structure was 76 in amino acid-supplemented cells and 185 in amino acid deprived cells (Fig. 3c).

To further clarify how amino acid starvation affects ULK1 clusters, we conducted the paircorrelation analysis. The pair-correlation magnitude, which quantifies the accumulation of molecules relative to a random distribution, was significantly higher in starved cells compared to fed cells at distances from $110 \mathrm{~nm}-300 \mathrm{~nm}$ (Fig. 3D). While the absolute difference in the pair correlation magnitude seems small, it is important to note that only a few percent of ULK1 molecules are induced by starvation to form high-order oligomers (Fig. 3c) and cause this change in pair-correlation. This result corroborates the observation that amino acid starvation induces the formation of larger structures with denser ULK1 molecules. 


\section{ULK1 structures containing Atg13 display variability in size, shape and multimeric state}

ULK1 interacts with Atg13 to regulate autophagy ${ }^{9-11}$. We therefore wondered whether the higherorder multimeric states of ULK1 in starved cells are related to the autophagy initiation complex containing both ULK1 and Atg13. To address this, we performed co-localization PALM experiments monitoring both ULK1 and Atg13. We transiently expressed Atg13 as a fusion with HaloTag in mEos2-ULK1-expressing HeLa cells. The HaloTag protein covalently binds organic fluorophores attached to a HaloTag ligand in a one to one ratio producing high photon counts (and thus high localization precision) ${ }^{41,42}$. We used the JF646 dye, which has no spectral overlap with mEos2, to generate PALM images (Fig 4a). In fed cells, both Atg13 and ULK1 formed small clusters that were distributed throughout the cytoplasm (Fig 4a, upper). Starved cells also contained small ULK1 clusters, many of which did not colocalize with Atg13. However, starvation induced large arc-shaped and spherical structures that contained both ULK1 and Atg13 (Fig. 4a, lower and Supplementary Fig. 4). ULK1 structures associated with Atg13 exhibited a variety of sizes, shapes, and multimeric states. Some of the structures contained small, dense ULK1 clusters, whereas some contained large ULK1 clusters with various oligomeric states (Fig. 4b). In contrast, ULK1 molecules that did not colocalize with Atg13 only formed small clusters (Fig. 4b, right). This co-localization PALM analysis showed that the clustering pattern is different between ULK1 structures that colocalized with Atg13 and those that did not colocalize with Atg13.

To further clarify the nature of ULK1 clusters that colocalize with Atg13, we performed cross-correlation analysis ${ }^{31,43}$ of ULK1 and Atg13. Like pair correlation analysis, cross-correlation analysis of two-color PALM data allows for robust quantification of co-distributions for two different proteins inside a cell ${ }^{37,43}$. When the two proteins are randomly distributed with respect to each other, the cross-correlation value becomes one. However, when two proteins interact and are more likely to co-localize within a short distance, the magnitude of the cross-correlation is larger than one up to this distance. We therefore used this technique to measure the extent of interaction between ATG13 and ULK1. In fed cells, we observed a peak of the cross-correlation at short distances up to $100 \mathrm{~nm}$; however, under starvation the peak was significantly higher and broader at longer distances (Fig. 4c). This result implies a more significant extent of starvation-induced co-localization between ULK1 and Atg13 in enlarged structures that might be relevant to the initiation of autophagy. 


\section{Starvation induces distinct Atg13-containing structures with high oligomeric states of}

\section{ULK1 molecules.}

In order to verify that the observed structures containing both Atg13 and ULK1 are involved in autophagy and to quantitatively analyze them, we isolated their localizations from the remainder of Atg13 and ULK1. Based on the previous cross-correlation analysis, we separately analyzed the ULK1 molecules that have a distance shorter than $100 \mathrm{~nm}$ to Atg13 (Atg13-bound structures) and those that have a distance longer than $100 \mathrm{~nm}$ (Atg13-free structures) (Fig. 5a and Materials and Methods). As in the previous quantification of the oligomeric state of ULK1 in Fig. 2 and Fig. 3, we determined the number of ULK1 molecules in each structure and in addition approximated their radius (Fig. 5b and Materials and Methods). In fed cells, ULK1 showed similar distribution patterns regardless of Atg13-bound states, exhibiting a maximum of 43 ULK1 molecules (Fig. 5b). There was also no detectable difference in the radii of the two types of ULK1 structures.

In contrast, amino acid starvation induced completely different patterns between the two types of ULK1 structures. Atg13-free structures had no detectable difference in their number of ULK1 molecules and in their radii compared to fed cells, whereas Atg13-bound structures contained significantly more ULK1 molecules up to 161 under starvation (Fig. 5b, Supplementary Fig. 4). To compare the abundance of structures having a large number of ULK1 molecules in fed and starved conditions, we set a threshold number of 30 ULK1 molecules. At this threshold, the difference in the distribution of the number of structures was most significant between fed and starved cells by various metrics (Supplementary Fig. 5). In short, if a lower threshold is set, the fraction of Atg13-bound structures in starved cells becomes smaller and so does its difference to fed cells. If a larger threshold is set, the number of Atg13-free structures in starved cells and the number of structures in fed cells becomes too small and the resulting large uncertainties prevent a meaningful statistical comparison. Starved cells had on average about 6-7 structures per cell that contained both Atg13 and more than 30 ULK1 molecules, which is a significant $\sim 18$-fold increase compared to fed cells (Fig 5c).

The normalized histogram of the radii of Atg13-free structures was roughly constant up to $\sim 200 \mathrm{~nm}$ in the radius, whereas Atg13-bound structures showed different distributions depending on the number of ULK1 molecules (Fig. 5d). When Atg13-bound structures contained less than 30 ULK1 molecules, the radii spanned a broad range from the smallest measurable values up to 300 $\mathrm{nm}$ and exhibited a peak at approximately $200 \mathrm{~nm}$ (Fig. 5d). When Atg13-bound structures 
contained more than 30 ULK1 molecules, the majority of radii was larger than $100 \mathrm{~nm}$ and radii up to $300 \mathrm{~nm}$ were detected. These results suggest that Atg13-bound structures with more than 30 ULK1 molecules tend to be larger compared to structures with less than 30 ULK1 molecules or structures without Atg13. To further characterize the density of ULK1 in these three classes of structures, we calculated the pair-correlation function, which quantifies the average density of neighboring ULK1 molecules relative to each ULK1 molecule at a given distance. Atg13-free structures formed smallest clusters with a low ULK1 density, whereas Atg13-bound structures with less than 30 ULK1 molecules formed the densest clusters (Fig. 5e). Interestingly, Atg13bound structures with more than 30 ULK1 molecules had a lower density but a tail up to long distances reflecting their larger size. Therefore, initially dense ULK1 clusters with Atg13 disintegrate to form larger Atg13-bound structures with a lower density of ULK1 molecules.

In summary, our analysis identified unique structures containing Atg13 and more than 30 ULK1 molecules that were induced by starvation. While only about $6.3 \%$ of all ULK1 molecules were found in these structures in starved cells (Fig. 5b), their abundance in starved cells was significantly higher ( $\sim 18$ fold) compared to fed cells. ULK1 clusters that did not associate with Atg13 had a lower density compared to Atg13-bound structures with less than 30 ULK1 molecules, which highlights the different state of ULK1 when associated with Atg13. The unique starvation induced structures that contain Atg13 and more than 30 ULK1 molecules exhibited a low ULK1 density, which might indicate disintegration of dense ULK1 clusters in these structures.

\section{Large ULK1 structures formed under starvation are in close proximity to the ER}

Given that several studies have shown that autophagy initiation occurs on the $\mathrm{ER}^{8,17}$, we wondered whether the starvation induced structures with more than 30 ULK1 molecules are associated with the ER. To address this, we performed co-localization PALM analysis using mEos2-ULK1 cells after the cells were transiently transduced to express HaloTag-tagged Sec61 $\beta$, a well-characterized ER marker. mEos2-ULK1 was localized not only on or near the tubular ER regions but also to some extent throughout the cytoplasm in both fed and starved cells (Fig. 6a). In starved cells, the large ULK1 clusters were found in contact or close to the ER (Fig. 6a, lower right). In order to determine the location of starvation induced structures with more than 30 ULK1 molecules with respect to the ER, we separately analyzed ULK1 molecules that appeared within a $100 \mathrm{~nm}$ distance from an ER localization as in our analysis with Atg13 in Fig. 5b. In fed cells, the distribution of 
the number of ULK1 molecules and the radii of structures was similar whether they were in proximity to the ER or not (Fig. 6b, left). Only in starved cells, we again observed structures with a high number of ULK1 molecules that were not found in fed cells. Importantly, all but one of these structures with more than 30 ULK1 molecules were located within a $100 \mathrm{~nm}$ distance from the ER (Fig. 6b, right and Supplementary Fig. 6).

To further visualize the distinct population of structures with a high oligomeric state of ULK1 at the ER, we created a normalized histogram of the number of ULK1 molecules in each structure (Fig. 6c). Structures that had a distance of more than $100 \mathrm{~nm}$ from an ER localization contained up to 40 ULK1 molecules, whereas structures closer than $100 \mathrm{~nm}$ to the ER contained up to 130 ULK1 molecules (Fig. 6c). Therefore, starvation induced structures that contain a high number of ULK1 molecules and that have earlier been shown to contain Atg13 (Fig. 5) are located at and near the ER. In both fed and starved cells, about $80 \%$ of all ULK1 molecules were within a distance of $100 \mathrm{~nm}$ from an ER localization whereas over 95\% of ULK1 molecules were within a distance of $800 \mathrm{~nm}$ (Fig. 5b). Therefore, no significant difference of the ULK1-localization with respect to the ER was observed upon starvation. These result suggests that ULK1 molecules likely engaging in autophagy are localized at and near the ER, where they accumulate to form larger structures of $\geq 30$ ULK1 molecules. These structures might develop into or be the phagophores in proximity to the ER.

\section{Discussion}

In this study, we have applied qPALM to characterize the behaviors of endogenous ULK1 during autophagy initiation at the single-molecule level with $20 \mathrm{~nm}$ spatial resolution. None of previous PALM studies using endogenously tagged proteins ${ }^{35-38}$ could not quantify the number of proteins due to the challenges caused by the blinking of PSFPs. Therefore, our study is the first that quantified the molecular number of endogenous proteins in cells at the nanoscopic length scale. This has provided unprecedented levels of insight into the hierarchical ordering of ULK1 oligomerization during autophagy induction.

The main principle of qPALM analysis is to group localizations from the same fluorophore within a spatio-temporal threshold ${ }^{26,27,44}$. A challenge for this quantification is that PSFPs do not emit at a constant intensity but rather exhibit a variable number of fluorescent bursts separated by 
a variable time. This phenomenon causes single PSFPs to appear as clusters with a variable number of localizations ${ }^{26,31,45,46}$. To quantify the oligomeric states of a protein of interest with this qPALM approach, the PSFP must meet two requirements: First, the PSFP must irreversibly bleach within a short time compared to the entire imaging sequence; second, the distribution of dark times between fluorescent bursts of single PSFP must be known. Since the photophysical properties of PSFPs strongly depend on the experimental conditions, we had to determine both spatio- and temporal thresholds for grouping localizations under our experimental condition. We performed PALM experiments at a very low $405 \mathrm{~nm}$ power to avoid the spatio-temporal overlap of PSFPs. This allowed us to determine the dark time distribution of mEos 2 and a dark-time cutoff of $4.5 \mathrm{~s}$, which covers more than $99 \%$ of ULK1 molecules in a single cell (Supplementary Fig. 2).

We found that ULK1 forms clusters with up to 76 molecules in fed conditions. Starvation induced a small fraction of ULK1 structures containing a higher number up to 185 ULK1 molecules. ULK1 that colocalized with Atg13 formed structures of small dense clusters as well as arc and spherical morphologies in starved conditions (Fig. 3 and 4). These Atg13-containing structures were not found in fed cells. ULK1 that did not colocalize with Atg13 was not able to form such highly dense clusters of ULK1, supporting the relevance of the structures to autophagy (Fig. 5b and 5e). The arc-shaped and large spherical structures contained high numbers of ULK1 molecules in various multimeric states (Fig. 5b) and formed only under starvation for ULK1 molecules that colocalized with Atg13. These arc and spherical structures of ULK1 might be relevant to the early phagophore structures described in a recent study by Karanasios et al. ${ }^{17}$. We therefore interpret these arc-shaped structures to be phagophores, which emerge from dense, small clusters containing both ULK1 and Atg13 and become spherical structures of large sizes. Although these structures contain the largest number of ULK1 molecules, they are less dense in ULK1, indicating disintegration of dense ULK1 clusters.

Our colocalization analysis revealed that ULK1 molecules in fed and starved cells are located not only at the ER but also throughout the whole cytoplasm. Distinctively among the different forms of ULK1 clusters, the starvation-induced Atg13-bound structures containing > 30 ULK1 molecules were found only on or near the ER. However, we do not exclude the possibility that other organelles, such as mitochondria, might have contacts with those ULK1 structures. This analysis revealed there might be a threshold number of ULK1 molecules around 30, which is necessary to drive the ULK1 structures to depart from the ER during autophagy induction. These 
structures then become less dense in ULK1 when growing and departing from the ER. Based on our result, we speculate that phagophores might form only when a critical number of 30 ULK1 molecules accumulate as a cluster.

The application of qPALM techniques to endogenously tagged ULK1 has provided unprecedented insights into the nature and the behavior of ULK1 molecules at the initiation of autophagosome formation. Whether the ULK1 clusters are part of phagophores or reflect membrane structures distinct from phagophore will need further investigation. Our study also could not clarify the molecular basis for how such large ULK1 clusters form and dynamically change in the multimeric states and morphology. The single molecule qPALM analysis could become useful in conjunction with genetic and biochemical approaches to address such outstanding questions in future studies.

\section{Materials and Methods}

\section{Generation of genome-edited cells}

To introduce the mEos2 tag into the ULK1 locus of the genome of HeLa cells, we used the CRISPR-Cas9-assisted genome editing technique ${ }^{47}$. The detail of the general procedure has been described in our previous report ${ }^{48}$. The gRNA sequence (GCGGCCGGGCTCCATGGCGC) was cloned into pSpCas9(BB)-2A-GFP (Addgene, PX458; deposited by Dr. Feng Zhang). The replacement DNA was designed to contain $700 \mathrm{bps}$ of the homologous region for both sides of the inserted mEos 2 sequence. Four tandem repeats of GGT nucleotide sequences were added to the end of the mEos 2 sequence. The gRNA plasmid and the replacement plasmid were introduced into HeLa cells using the Neon transfection system (ThermoFisher Scientific). GFP-positive cells were sorted and plated in 96-well plates as a single cell. Individual clones were screened for expression of mEos2-ULK1 by western blotting and confirmed by genomic DNA sequencing.

\section{DNA construction}

mEos2-tagged ULK1 construct and HaloTag tagged Atg13 and Sec61 $\beta$ constructs were prepared by PCR amplification and cloned into pCSII-mEos2 and pCSII-HaloTag plasmids derived from the pCSII-EF vector (provided by Dr. Nik Somia, University of Minnesota). Restriction enzyme 
sites at XhoI and NotI in the vector were used to clone mEos2 and HaloTag. These pairs of primers were used to clone the genes: 5'-gagcgagtcgaccatgagtgcgattaagcca-3' and 5'gagcactcgagccacctccgecacctcgtctggcattgtcag-3' for mEos2;

5 '-gagcgactcgagcaccatggcagaaatcggtact-3' and 5'-gtctcgaagcggccgcattgcatacgatggtcgacccggaaatctccagcgttgacagcca-3' for HaloTag. The genes of ULK1, Atg13 and Sec61 $\beta$ were cloned into the mEos2 and HaloTag plasmids at SalI and NotI sites. The LC3B plasmid (Addgene \#11546) was a kind gift from Dr. Karla Kirkegaard.

\section{Co-immunoprecipitation and western blotting}

The mEos2-tagged endogenous ULK1 was enriched by immunoprecipitation using an anti-ULK1 antibody (Santa Cruz Biotech., sc-10900) following the procedure we described in our previous report ${ }^{10,20,48}$. We used a lysis buffer containing $40 \mathrm{mM}$ HEPES, pH 7.4, $120 \mathrm{mM} \mathrm{NaCl}, 1 \mathrm{mM}$ EDTA, $50 \mathrm{mM} \mathrm{NaF}, 1.5 \mathrm{mM} \mathrm{Na}_{3} \mathrm{VO}_{4}, 10 \mathrm{mM}$ beta-glycerophosphate, 1\% Triton X-100 (SigmaAldrich, X100) supplemented with protease inhibitors (Roche, 05056489001). Immunoprecipitated proteins were run on Tris-glycine gels (ThermoFisher Scientific, XP04125BOX), transferred onto immunoblot polyvinylidene difluoride (PVDF) membranes (Bio-

Rad, 1620177), and detected using ECL reagents (GenDEPOT, 20-300B). The following antibodies were used for western blotting: ULK1 (sc-33182) from Santa Cruz Biotech.; p-Atg14 Ser29 (13155) and Atg14 (96752) from Cell Signaling Technology; Atg13 antibody was described in our previous report ${ }^{10}$.

\section{Preparation of cells for imaging.}

HeLa cells were maintained in fluorobrite DMEM (Invitrogen) supplemented with 10\% fetal bovine serum, $4 \mathrm{mM}$ glutamine (Gibco), $1 \mathrm{mM}$ sodium pyruvate (Gibco) and 1\% penicillinstreptomycin antibiotics (Invitrogen) in a T25 flask. Cells were subcultured on eight-well chambered cover glasses (Cellvis, C8-1.58-N) $24 \mathrm{~h}$ before transfection. Transient transfections were performed using GeneJET (ThermoFisher Scientific) 12-24 h before the measurements. The transfected cells were cultured in full medium or Earle's Balanced Salt Solution (EBSS) for 150 min in the presence of $180 \mathrm{nM}$ bafilomycin A1 (BAFA1). BAFA1 is widely used in the field to increase the number of detected autophagosomes in each cell by inhibiting the fusion of autophagosomes with lysosomes. All the imaging experiments have been performed in fixed cells. 
For fixation, cells were treated with $3 \%(\mathrm{v} / \mathrm{v})$ formaldehyde for $30 \mathrm{~min}$. After fixation, we used Dulbecco's phosphate-buffered saline (PBS) with calcium and magnesium (Gibco) for measurements.

For two-color imaging of mEos2-ULK1 with Atg13-HaloTag or the ER marker Sec61HaloTag, we used the organic dyes Oregon green (OG) and JF 646 conjugated to the HaloTag ligands (Promega). Both dyes exhibit no overlap of their absorption and emission spectra with photoconverted mEos 2 . The dyes were added to live cells on the coverglass of the 8 -well chambers at a final concentration of $500 \mathrm{nM}$ for OG and 1 to $10 \mathrm{nM}$ for JF 646. After an incubation time of 15-20 min cells were fixed with 3\% formaldehyde and washed five times with PBS containing 1\% Triton-X (TX-100) to remove access dyes that did not covalently bind to the HaloTag.

\section{PALM and conventional fluorescence data acquisition}

All microscopy experiments were performed with a Nikon Ti-E inverted microscope with a Perfect Focus System and recorded on an electron-multiplying CCD camera (Ixon89U1tra DU897U; Andor) at a frame rate of $20 \mathrm{~Hz}$ as described in detail in the supplemental information. In short, for PALM imaging of mEos2-ULK1 one photoactivation frame (405 nm laser, 1-251 $\mu \mathrm{W}$ corresponding to a power density of roughly $0.06-15 \mathrm{~W} / \mathrm{cm}^{2}$ ) with simultaneous transmitted light imaging was followed by nine frames of excitation $(561 \mathrm{~nm}, 17 \mathrm{~mW}$ corresponding to a power density of roughly $1 \mathrm{~kW} / \mathrm{cm}^{2}$ ). For two-color PALM imaging of mEos2-ULK1 and HaloTag JF646, the excitation frames consisted of five $561 \mathrm{~nm}$ excitation frames for $\mathrm{mEos} 2$ followed by five 640 $\mathrm{nm}$ excitation frames $\left(640 \mathrm{~nm}, 17.5 \mathrm{~mW}\right.$, roughly $\left.1 \mathrm{~kW} / \mathrm{cm}^{2}\right)$. Photoactivation and excitation cycles were repeated until all mEos2/JF646 molecules were imaged and bleached typically until a movie recorded at $20 \mathrm{~Hz}$ reached 8,000-15,000 frames. The point spread functions of single molecules were then fitted with an elliptical Gaussian function using Insight3 (Zhuang lab, http://zhuang.harvard.edu/software.html) to determine their centroid positions, intensities, widths, and ellipticities. For all localizations, the $\mathrm{x}$ and $\mathrm{y}$ coordinates, photon number, background photons, width, the frame of appearance, and other fit parameters were saved in a molecule list for further analysis. To correct for drift, all molecule lists were drift corrected using the repetitive transmitted light images ${ }^{49}$. The conventional fluorescence images were generated by averaging the 50 frames (Fig. S1) and 500 frames for Fig. 3A recorded at $20 \mathrm{~Hz}$ with a $488 \mathrm{~nm}$ excitation power density of $0.085 \mathrm{~W} / \mathrm{cm}^{2}$. 


\section{Quantitative analysis of molecule numbers}

Once a single mEos2 molecule is stochastically photoactivated, it emits a variable number of fluorescent bursts that are separated by a variable dark time until irreversible bleaching occurs. These fluorescent bursts can be combined since the $405 \mathrm{~nm}$ photoactivation rate was kept low enough to avoid spatio-temporal overlap with a nearby mEos 2 molecule. The combination of these fluorescent bursts is based on two thresholds: 1) a distance threshold that specifies the maximum separation of fluorescent bursts from a mEos2 molecule (corresponding to the localization precision) and 2) a temporal dark-time threshold that specifies the maximum time between fluorescent bursts from a mEos 2 molecule. The distance threshold was measured using the paircorrelation function (Suppl. Fig. S2D), which revealed that fluorescent bursts from single mEos2 molecules are spatially separated by less than $80 \mathrm{~nm}$. The dark-time threshold was determined from the cumulative dark-time histogram of fluorescent bursts and set to 4.5 seconds, which covers 99\% of the dark times (Suppl. Fig S2E and F). Based on these two parameters, a custom written Matlab procedure combines two localizations from fluorescent bursts to a photon-weighted average position if they are separated by less than $80 \mathrm{~nm}$ and less than $4.5 \mathrm{~s}$. If a third localization again appears within the cutoff time with respect to the preceding one and within $80 \mathrm{~nm}$ compared to the averaged position of the previous two localizations, a new photon-weighted average position is calculated from the three localizations. This iterative process is repeated until all localizations from the same mEos 2 molecules are combined to a photon weighted average position. The average position has a higher localization precision due to the increased number of photons. From the resulting blink-corrected molecule list, the actual number of mEos 2 molecules and their spatial distribution can be further analyzed.

To count the number of ULK1 molecules in initiation complexes and forming autophagosomal structures in an unbiased way, we determined their size distribution in the amino acid starved conditions using the pair-correlation function of the blink-corrected mEos 2 molecule list (Fig. 3D). The pair correlation function $\mathrm{g}(\mathrm{r})$ was above 1 outside the experimental error (standard error of mean) up to a distance of $800 \mathrm{~nm}$, indicating accumulation of ULK1 molecules beyond a random distribution. Therefore, ULK1 molecules within a radius of $400 \mathrm{~nm}$ were assigned to one structure or cluster. Structures and clusters with a certain number of ULK1 molecules were then accumulated in a histogram with bin width 10 . 


\section{Alignment of two-color PALM data.}

To superimpose two color PALM data for images or cross correlation analysis, we recorded conventional fluorescence images of fluorescent beads (TetraSpeck microspheres, Invitrogen T7279) that were excited at $561 \mathrm{~nm}$ and $640 \mathrm{~nm}$ and appeared in both channels. The transformation between the two channels across the field of view was then obtained by localizing individual beads in both channels and by determining the 3rd order polynomial transformation between their coordinates. The accuracy of the transformation was then verified by recording a different fields of view of fluorescent beads and superimposing both channels using the determined transformation. As seen in Suppl. Fig. S4, the deviation of the bead locations from both channels was less than 19 $\mathrm{nm}$, which is smaller than the localization precision of PALM.

\section{Spatial Cross-Correlation analysis and cluster identification}

After identification of blink corrected ULK1 molecules, molecules were assigned to clusters. The radial distribution (or pair correlation) among all blink corrected molecules was calculated and molecules that were within $400 \mathrm{~nm}$ of each other were identified as ULK1 clusters. The obtained partial radial distribution function was normalized by the number of molecules in both datasets and the area of the field of view ${ }^{31}$. Next, a spatial cross-correlation between the blink corrected ULK1 molecules and Atg13 localizations was performed to determine the distance distribution between the two proteins. Due to the large number of localizations in both the blink corrected ULK1 and the Atg13 localization datasets, we set a $2 \mu \mathrm{m}$ cutoff in the nearest neighbor search, which only requires 10-30 GB of working memory. This method allows for the identification of molecule pairs within a specified distance distribution across the entire desired field of view for many movies using available computational resources (analysis code was written in MATLAB 2018b and run on an Alienware Aurora G6 computer with $3.80 \mathrm{GHz}$ CPU, $40 \mathrm{~GB}$ of working memory, NVIDIA GeForce GTX 10708 GB graphics card, and 900 GB of disk space). Next, ULK1 clusters that contained at least one ULK1 molecule within $100 \mathrm{~nm}$ of an Atg13 localization (labeled "with Atg13") were segregated from ULK1 clusters with no molecules within $100 \mathrm{~nm}$ of an Atg13 localizations (labeled "without Atg13"). Cluster properties such as the radius and the number of ULK1 molecules were determined separately for a comparison of fed 
and starved cells. This cross-correlation analysis was also applied to ULK1 molecules and ER localizations.

\section{Estimating radii of structures:}

To estimate the radius of the ULK1 clusters, the $\mathrm{x}$ and $\mathrm{y}$ positions of the molecules inside the cluster were averaged to find the center and the distance between each molecule and the center was calculated. The mean of the distances from the center was then used to approximate the radius of the ULK1 cluster.

\section{Acknowledgment:}

Research reported in this publication was supported by the National Institute of General Medical Sciences of the National Institutes of Health under Award number R21GM127965 (E.M.P) and R35GM130353 (D.H.K). We thank Yu Xu for her valuable help and input in the blink correction code. 


\section{References}

1 Levine, B. \& Kroemer, G. Autophagy in the pathogenesis of disease. Cell 132, 27-42 (2008).

2 Mizushima, N. \& Levine, B. Autophagy in mammalian development and differentiation. Nature cell biology 12, 823-830 (2010).

3 Noda, T. \& Ohsumi, Y. Tor, a phosphatidylinositol kinase homologue, controls autophagy in yeast. Journal of Biological Chemistry 273, 3963-3966 (1998).

4 Kamada, Y., Sekito, T. \& Ohsumi, Y. in TOR 73-84 (Springer, 2004).

5 Jung, C. H., Ro, S.-H., Cao, J., Otto, N. M. \& Kim, D.-H. mTOR regulation of autophagy. FEBS letters 584, 1287-1295 (2010).

6 Hailey, D. W. et al. Mitochondria supply membranes for autophagosome biogenesis during starvation. Cell 141, 656-667 (2010).

7 Hamasaki, M. et al. Autophagosomes form at ER-mitochondria contact sites. Nature 495, 389-393 (2013).

8 Biazik, J., Ylä-Anttila, P., Vihinen, H., Jokitalo, E. \& Eskelinen, E.-L. Ultrastructural relationship of the phagophore with surrounding organelles. Autophagy 11, 439-451 (2015).

9 Ganley, I. G. et al. ULK1 - ATG13 - FIP200 complex mediates mTOR signaling and is essential for autophagy. Journal of Biological Chemistry 284, 12297-12305 (2009).

10 Jung, C. H. et al. ULK-Atg13-FIP200 complexes mediate mTOR signaling to the autophagy machinery. Molecular biology of the cell 20, 1992-2003 (2009).

11 Hosokawa, N. et al. Nutrient-dependent mTORC1 association with the ULK1-Atg13FIP200 complex required for autophagy. Molecular biology of the cell 20, 1981-1991 (2009).

12 Mercer, C. A., Kaliappan, A. \& Dennis, P. B. A novel, human Atg13 binding protein, Atg101, interacts with ULK1 and is essential for macroautophagy. Autophagy 5, 649-662 (2009).

13 Hara, T. et al. FIP200, a ULK-interacting protein, is required for autophagosome formation in mammalian cells. The Journal of cell biology 181, 497-510 (2008).

14 Chang, Y.-Y. \& Neufeld, T. P. An Atg1/Atg13 complex with multiple roles in TORmediated autophagy regulation. Molecular biology of the cell 20, 2004-2014 (2009).

15 Chan, E. Y. W., Longatti, A., McKnight, N. C. \& Tooze, S. A. Kinase-inactivated ULK proteins inhibit autophagy via their conserved C-terminal domains using an Atg13independent mechanism. Molecular and cellular biology 29, 157-171 (2009).

16 Lamb, C. A., Yoshimori, T. \& Tooze, S. A. The autophagosome: origins unknown, biogenesis complex. Nature reviews Molecular cell biology 14, 759-774 (2013).

17 Karanasios, E. et al. Autophagy initiation by ULK complex assembly on ER tubulovesicular regions marked by ATG9 vesicles. Nature communications 7, 1-17 (2016).

18 Itakura, E. \& Mizushima, N. Characterization of autophagosome formation site by a hierarchical analysis of mammalian Atg proteins. Autophagy 6, 764-776 (2010).

19 Karanasios, E. et al. Dynamic association of the ULK1 complex with omegasomes during autophagy induction. Journal of cell science 126, 5224-5238 (2013). 
20 Park, J.-M. et al. The ULK1 complex mediates MTORC1 signaling to the autophagy initiation machinery via binding and phosphorylating ATG14. Autophagy 12, 547-564 (2016).

21 Betzig, E. et al. Imaging intracellular fluorescent proteins at nanometer resolution. Science 313, 1642-1645 (2006).

22 Shroff, H., White, H. \& Betzig, E. Photoactivated localization microscopy (PALM) of adhesion complexes. Curr Protoc Cell Biol Chapter 4, Unit 4.21, doi:10.1002/0471143030.cb0421s41 (2008).

23 Annibale, P., Vanni, S., Scarselli, M., Rothlisberger, U. \& Radenovic, A. Quantitative photo activated localization microscopy: unraveling the effects of photoblinking. PloS one 6, e22678 (2011).

24 Gunzenhäuser, J., Olivier, N., Pengo, T. \& Manley, S. Quantitative super-resolution imaging reveals protein stoichiometry and nanoscale morphology of assembling HIVGag virions. Nano letters 12, 4705-4710 (2012).

25 Lee, S.-H., Shin, J. Y., Lee, A. \& Bustamante, C. Counting single photoactivatable fluorescent molecules by photoactivated localization microscopy (PALM). Proceedings of the National Academy of Sciences 109, 17436-17441 (2012).

26 Puchner, E. M., Walter, J. M., Kasper, R., Huang, B. \& Lim, W. A. Counting molecules in single organelles with superresolution microscopy allows tracking of the endosome maturation trajectory. Proceedings of the National Academy of Sciences 110, 1601516020 (2013).

27 Durisic, N., Laparra-Cuervo, L., Sandoval-Álvarez, Á., Borbely, J. S. \& Lakadamyali, M. Single-molecule evaluation of fluorescent protein photoactivation efficiency using an in vivo nanotemplate. Nature methods 11, 156-162 (2014).

28 Van Lengerich, B., Agnew, C., Puchner, E. M., Huang, B. \& Jura, N. EGF and NRG induce phosphorylation of HER3/ERBB3 by EGFR using distinct oligomeric mechanisms. Proceedings of the National Academy of Sciences 114, E2836-E2845 (2017).

29 Spahn, C. K. et al. A toolbox for multiplexed super-resolution imaging of the E. coli nucleoid and membrane using novel PAINT labels. Scientific reports 8, 1-12 (2018).

30 Sengupta, P. et al. Probing protein heterogeneity in the plasma membrane using PALM and pair correlation analysis. Nature methods 8, 969-975 (2011).

31 Veatch, S. L. et al. Correlation functions quantify super-resolution images and estimate apparent clustering due to over-counting. PloS one 7, e31457 (2012).

32 Erdmann, R. S. et al. Labeling strategies matter for super-resolution microscopy: a comparison between HaloTags and SNAP-tags. Cell chemical biology 26, 584-592 (2019).

33 Ripaud, L. et al. Overexpression of Q-rich prion-like proteins suppresses polyQ cytotoxicity and alters the polyQ interactome. Proceedings of the National Academy of Sciences 111, 18219-18224 (2014).

34 Gibson, T. J., Seiler, M. \& Veitia, R. A. The transience of transient overexpression. Nature methods 10, 715-721 (2013).

35 Cho, W.-K. et al. Super-resolution imaging of fluorescently labeled, endogenous RNA Polymerase II in living cells with CRISPR/Cas9-mediated gene editing. Scientific reports 6, 35949 (2016). 
36 Khan, A. O., Simms, V. A., Pike, J. A., Thomas, S. G. \& Morgan, N. V. CRISPR-Cas9 mediated labelling allows for single molecule imaging and resolution. Scientific reports 7, 1-9 (2017).

37 Hansen, A. S., Pustova, I., Cattoglio, C., Tjian, R. \& Darzacq, X. CTCF and cohesin regulate chromatin loop stability with distinct dynamics. Elife 6, e25776 (2017).

38 Khan, A. O. et al. Optimised insert design for improved single-molecule imaging and quantification through CRISPR-Cas9 mediated knock-in. Scientific reports 9, 1-13 (2019).

39 Cong, L. et al. Multiplex genome engineering using CRISPR/Cas systems. Science 339, 819-823 (2013).

40 Shcherbakova, D. M., Sengupta, P., Lippincott-Schwartz, J. \& Verkhusha, V. V. Photocontrollable fluorescent proteins for superresolution imaging. Annual review of biophysics 43, 303-329 (2014).

41 Benink, H. A. \& Urh, M. in Site-Specific Protein Labeling 119-128 (Springer, 2015).

42 England, C. G., Luo, H. \& Cai, W. HaloTag technology: a versatile platform for biomedical applications. Bioconjugate chemistry 26, 975-986 (2015).

43 Stone, M. B. \& Veatch, S. L. Steady-state cross-correlations for live two-colour superresolution localization data sets. Nature communications 6, 1-10 (2015).

44 Adhikari, S., Moscatelli, J., Smith, E. M., Banerjee, C. \& Puchner, E. M. Single-molecule localization microscopy and tracking with red-shifted states of conventional BODIPY conjugates in living cells. Nature communications 10, 1-12 (2019).

45 Annibale, P., Vanni, S., Scarselli, M., Rothlisberger, U. \& Radenovic, A. Identification of clustering artifacts in photoactivated localization microscopy. Nature methods $\mathbf{8 , 5 2 7}$ (2011).

46 Berardozzi, R., Adam, V., Martins, A. \& Bourgeois, D. Arginine 66 controls dark-state formation in green-to-red photoconvertible fluorescent proteins. Journal of the American Chemical Society 138, 558-565 (2016).

47 Hsu, P. D., Lander, E. S. \& Zhang, F. Development and applications of CRISPR-Cas9 for genome engineering. Cell 157, 1262-1278 (2014).

48 Park, J.-M. et al. ULK1 phosphorylates Ser30 of BECN1 in association with ATG14 to stimulate autophagy induction. Autophagy 14, 584-597 (2018).

49 McGorty, R., Kamiyama, D. \& Huang, B. Active microscope stabilization in three dimensions using image correlation. Optical nanoscopy 2, 3 (2013). 
a

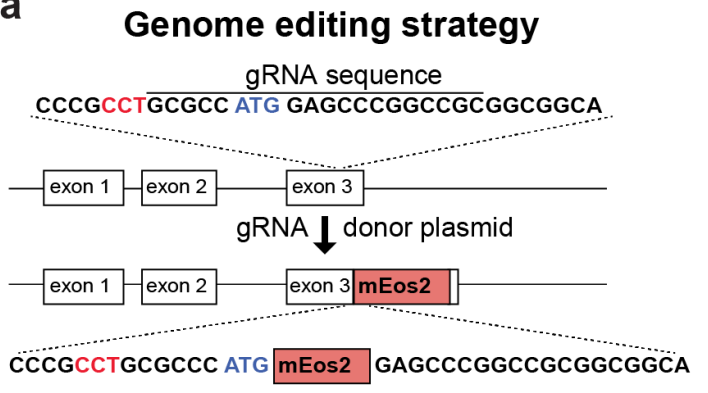

b
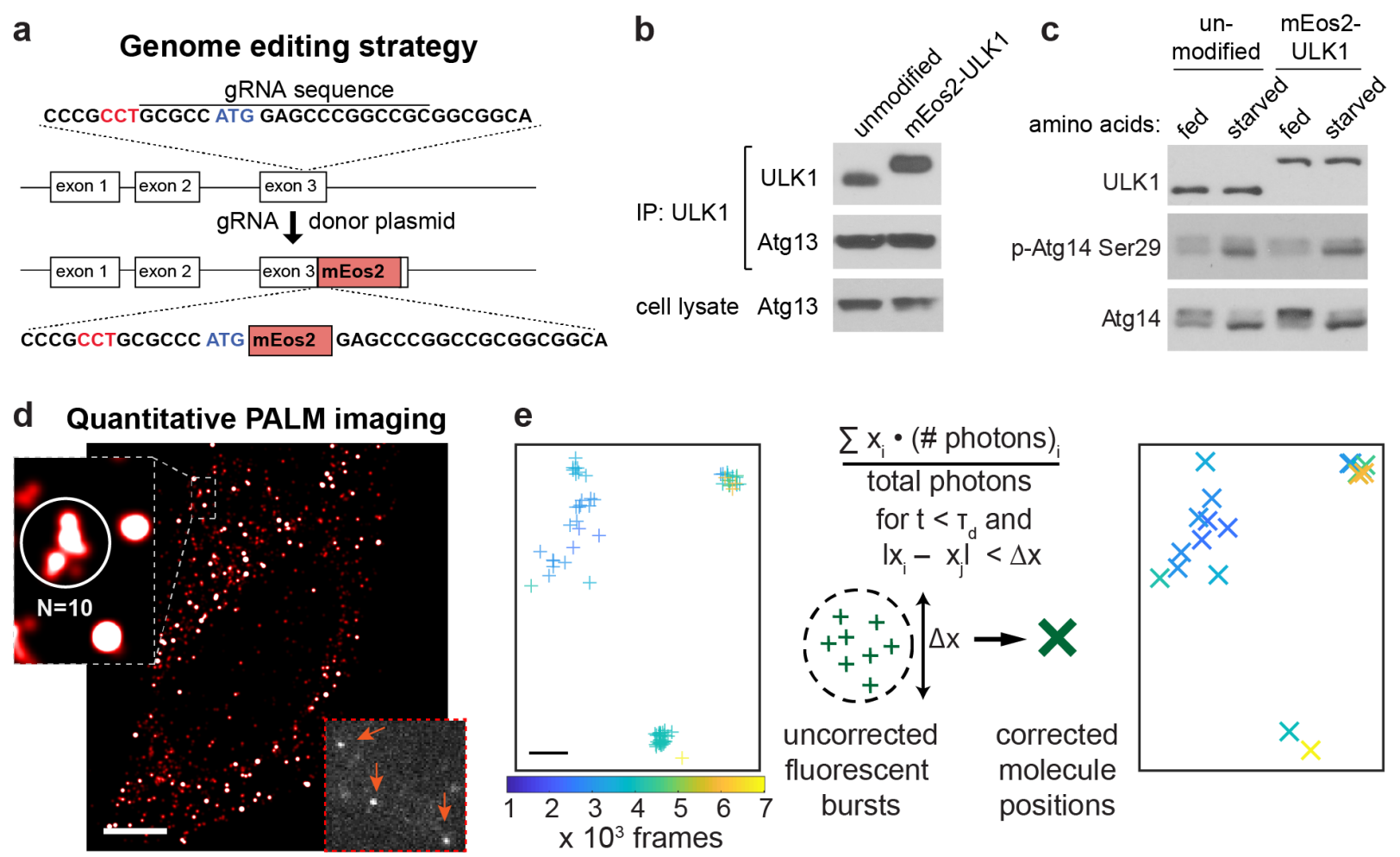

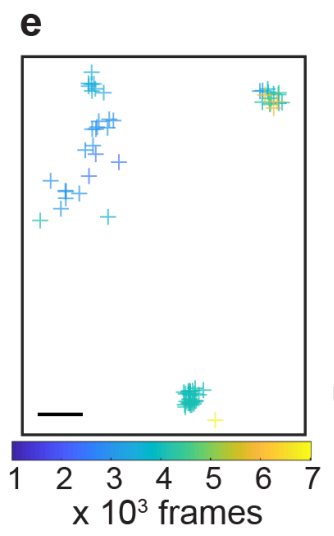

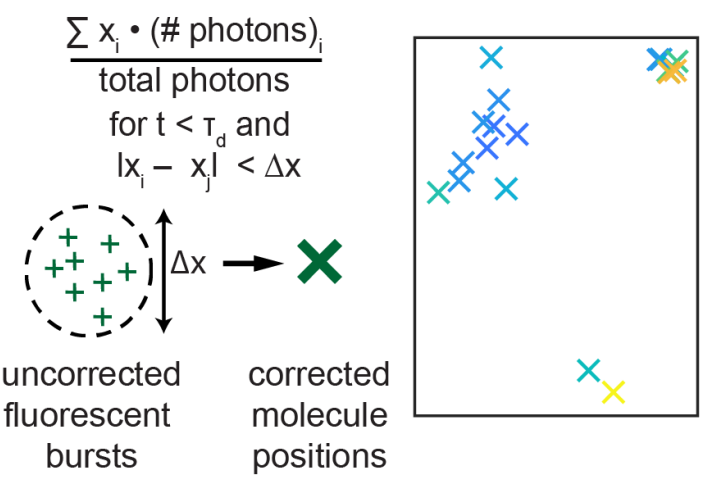

Fig. 1. Quantifying the nanoscopic distribution and oligomeric state of endogenous ULK1 with qPALM. a Schematics of endogenous tagging of ULK1 with mEos2. The PAM sequence of gRNA is depicted in red. The detailed procedure is described in the Materials and Method section. b The endogenously tagged ULK1 interacts with Atg13 to the same degree as the untagged endogenous ULK1. Immunoprecipitates were obtained from genome-modified HeLa cells (mEos2-ULK1) or unmodified HeLa cells using an anti-ULK1 antibody, and analyzed by western blotting for endogenous ULK1 and Atg13. c The endogenously tagged ULK1 is functionally intact for the activation of ULK1 in response to amino acid starvation. Cells were incubated in amino acid enriched full medium (fed) or amino acid deprived medium (starved) for $60 \mathrm{~min}$. The degree of phosphorylation of Atg14 Ser29, an ULK1 target site ${ }^{20}$, was analyzed by western blotting. d PALM image (red/white, scale bar $5 \mu \mathrm{m}$ ) showing the nanoscopic distribution of ULK1 oligomers throughout the cytoplasm. The bottom right inset shows bright single-molecule fluorescence of mEos2-ULK1 in a single data acquisition frame. The zoom on the left exemplifies quantification of the size and oligomeric state of a ULK1 cluster. e Left: individual single-molecule localizations are shown as crosses with a color code according to their frame of appearance. Some mEos 2 molecules only appear in one frame and irreversibly bleach, whereas others blink multiple times and cause a cluster of localizations. Scale bar: $200 \mathrm{~nm}$. Right: After averaging localizations appearing within the determined dark time cut-off and within the localization uncertainty, blinking of mEos 2 was corrected and the number of ULK1 molecules was obtained. 

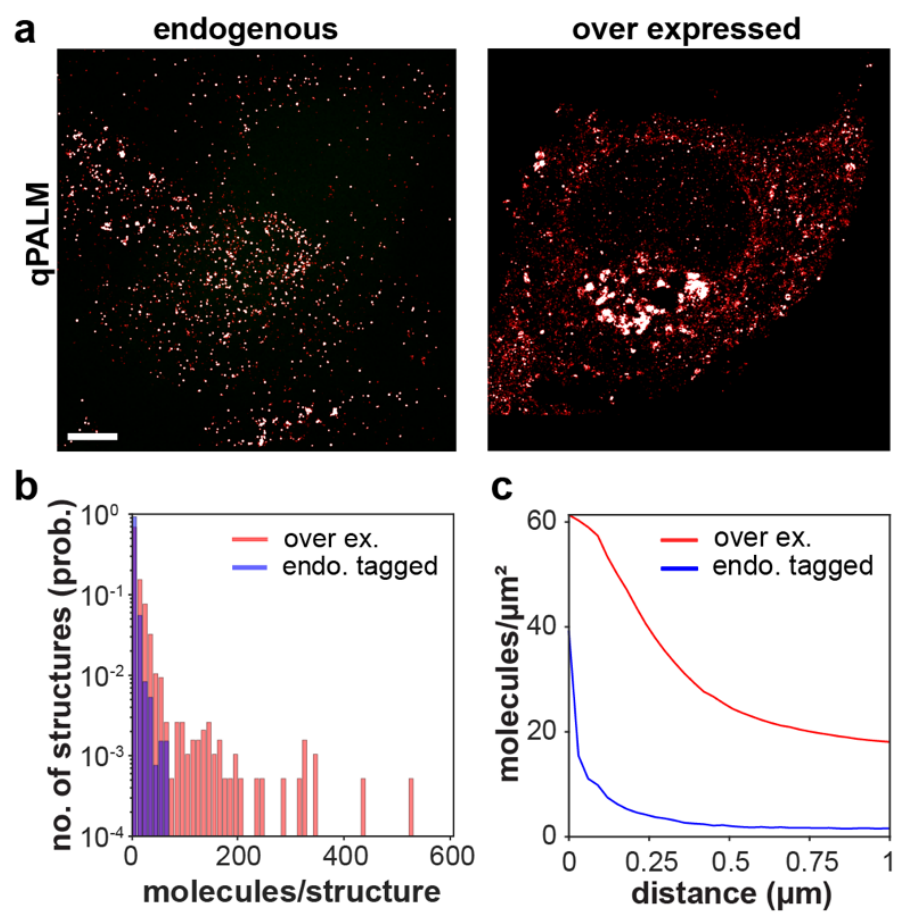

Fig.2. Endogenous tagging of ULK1 is required to avoid artifacts in quantifying the nanoscopic distribution and oligomeric state of ULK1. a qPALM images of endogenously tagged mEos2-ULK1 and overexpressed mEos2-ULK1 show that the overexpressed ULK1 forms highly dense structures, which might be clustering artifacts. Scale bar: $5 \mu \mathrm{m}$. b Analysis of qPALM data shows that overexpressed mEos2-ULK1 results in significantly more molecules and larger oligomers (red) than endogenously tagged mEos2-ULK1 (blue). ULK1-mEos2 molecules appearing within a distance of $400 \mathrm{~nm}$ were assigned to a structure, and a normalized histogram of the number of molecules per structure with bin width 10 was created. c Radial distribution comparison between overexpressed and endogenously tagged ULK1 highlights differences in degree of ULK1 protein clustering. Overexpressed cells contain larger cluster and higher ULK1 molecule densities when compared with endogenously tagged ULK1 clusters. 

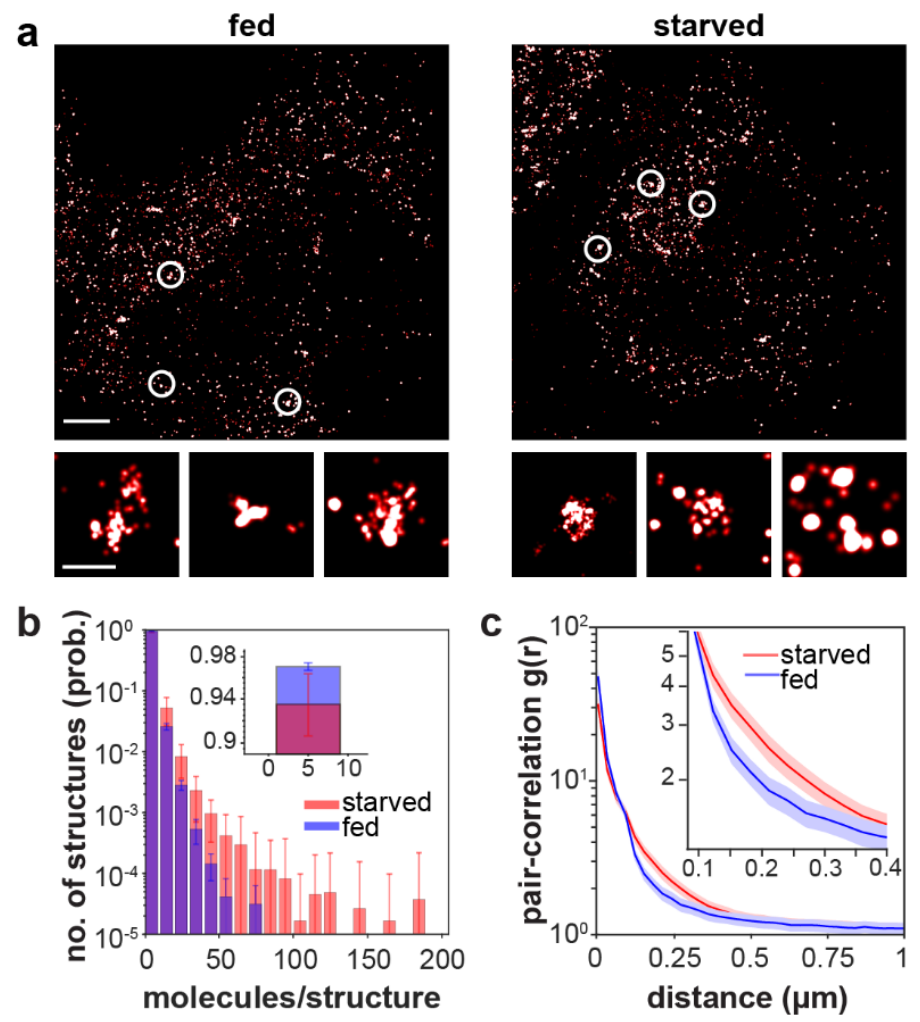

Fig. 3. Amino acid starvation induces clusters with more ULK1 molecules and larger size. a PALM images of mEos2-ULK1 in amino acid supplemented cells (fed) and amino acid deprived cells (starved). Scale bars: $5 \mu \mathrm{m}$. Magnified regions show small diffraction-limited structures, which were impossible to be resolved in conventional fluorescence images. Scale bar: $500 \mathrm{~nm}$. b The cluster analysis after the blink correction shows that amino acid starvation induced more higher-order oligomers and structures containing up to 185 ULK1 molecules (red) compared to 76 ULK1 molecules in fed cells (blue). The insert shows the top of the first bin to visualize that the majority of ULK1 is in oligomers with 1-10 molecules. The plot was generated by averaging the histogram of 19 fed and 23 starved cells, respectively. Error bars of each bin represent the standard error of the mean (SEM) with a variance calculated from the cell-to-cell variability. c Averaged pair correlation function analysis of the cells from $b$ and magnified region with statistically significant differences displays the accumulation of molecules with respect to a random distribution. The statistically significant difference at distances between $110 \mathrm{~nm}$ and $300 \mathrm{~nm}$ reflects and increase of cluster sizes in starved cells (red) compared to fed cells (blue). The absolute difference between fed and starved cells is small since only the small fraction of starvation induced high-order oligomers from Fig. $3 c$ contribute to the. The error band represent SEM with a variance calculated from the cell-to-cell variability. 
a
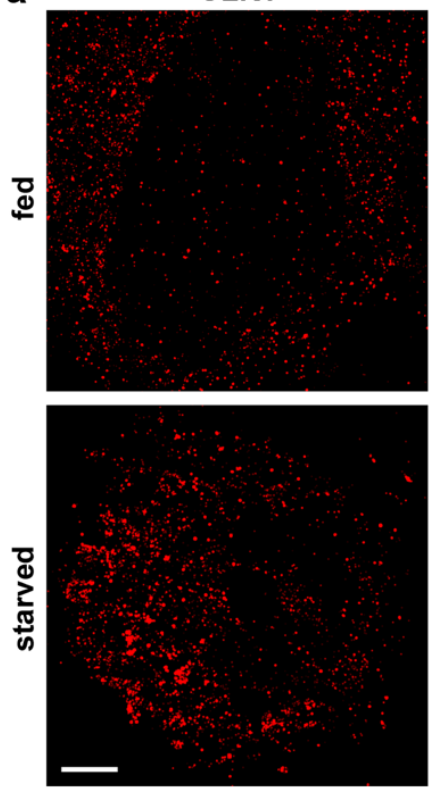

b
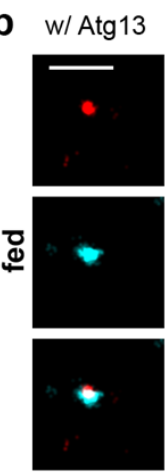
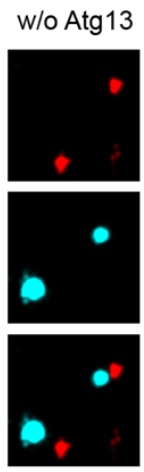

ULK1
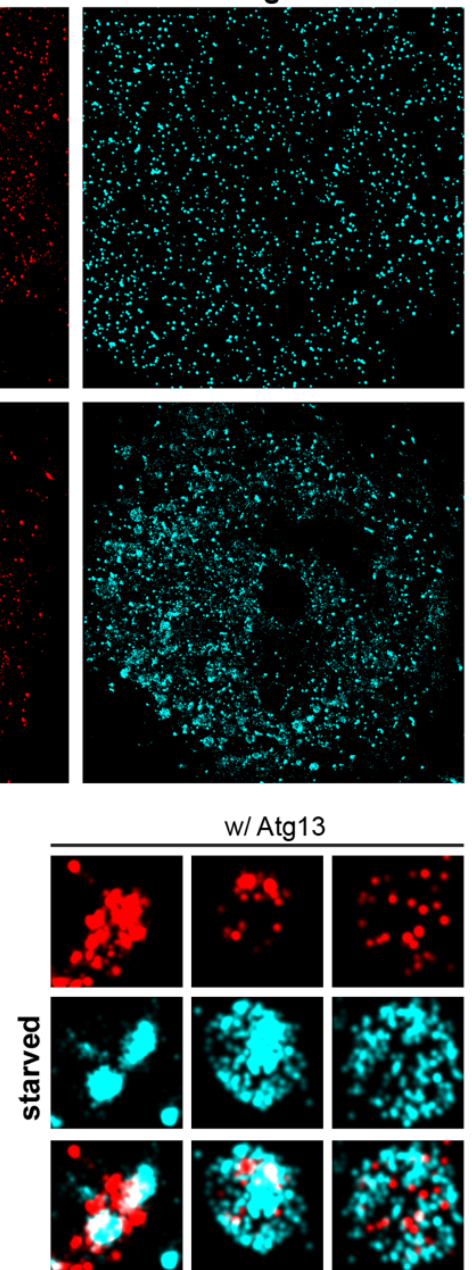

w/ Atg13

Atg13
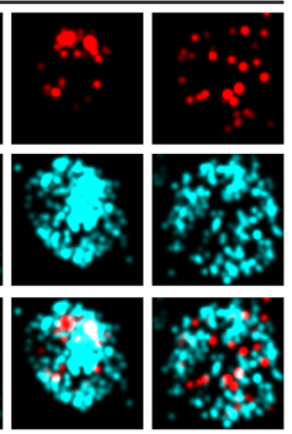

ULK1/Atg13
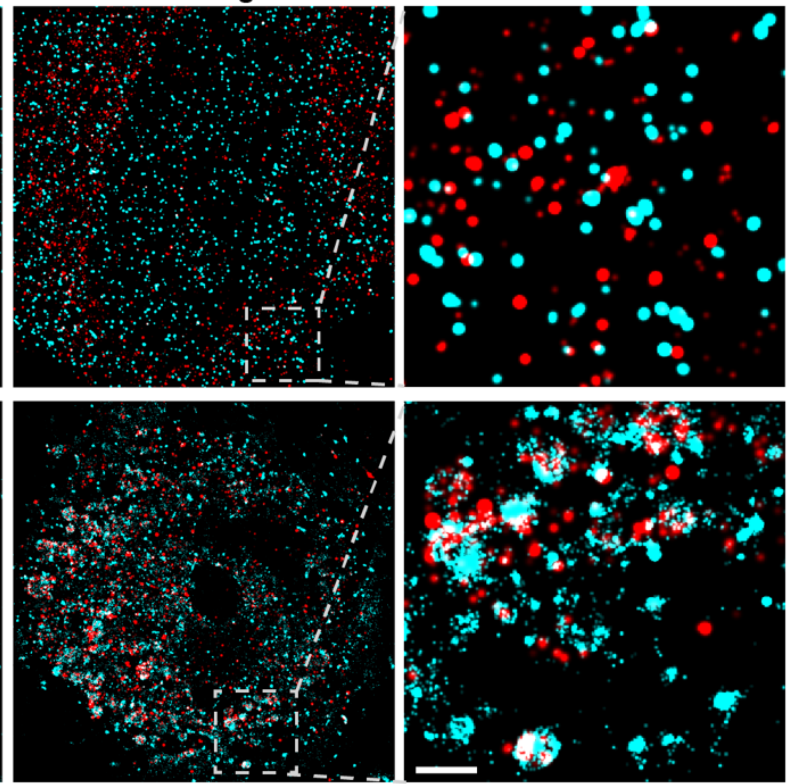

W/o Atg13

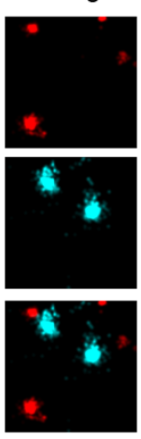

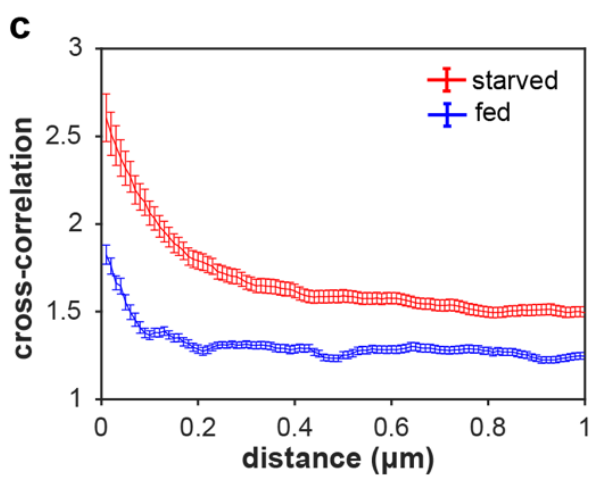

Fig. 4. ULK1 clusters containing Atg13 display variability in size, shape, and multimeric state under starvation. a Left: Two-color overlay of PALM images of mEos2-ULK1 (red) and HaloTag-Atg13 bound to JF646 (cyan) in fed (top) and starved cells (bottom). Scale bar: $5 \mu \mathrm{m}$. Right: Magnified images show the presence of various small dense ULK1 and Atg13 clusters that do or do not co-localize (upper). Upon starvation, larger spherical and arc-shaped structures containing both Atg13 and ULK1 become visible (lower). Scale bar, $1 \mu \mathrm{m}$. b Left: Examples of magnified two-color PALM images in fed cells show small ULK1 structures that do not co-localize with Atg13. Right: In starved cells Atg13 forms highly dense clusters and larger arc-shaped or spherical structures that are associated with ULK1. ULK1 that does not contain Atg13 only forms small cluster. Scale bar: $500 \mathrm{~nm}$. c Cross-correlation of ULK1 and Atg13 shows colocalization up to a distance of $100 \mathrm{~nm}$ between ULK1 and Atg13 in fed cells (blue) and a significantly larger degree of co-localization beyond $200 \mathrm{~nm}$ in starved cells (red). The error bar corresponds to SEM from seven fed and ten starved cells. 
a all localizations

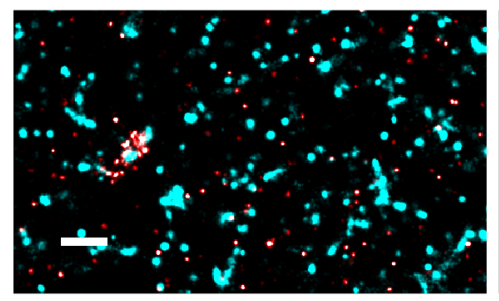

\section{b}

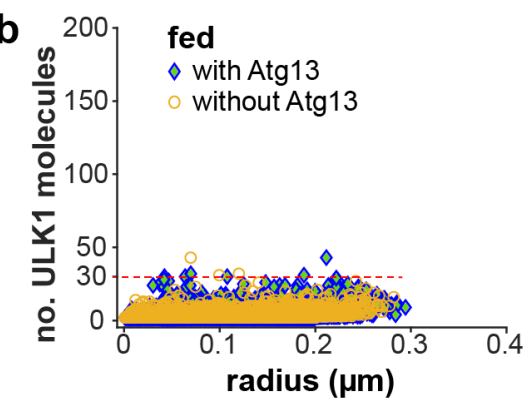

$\mid$ ULK1-Atg13| $100 \mathrm{~nm}$
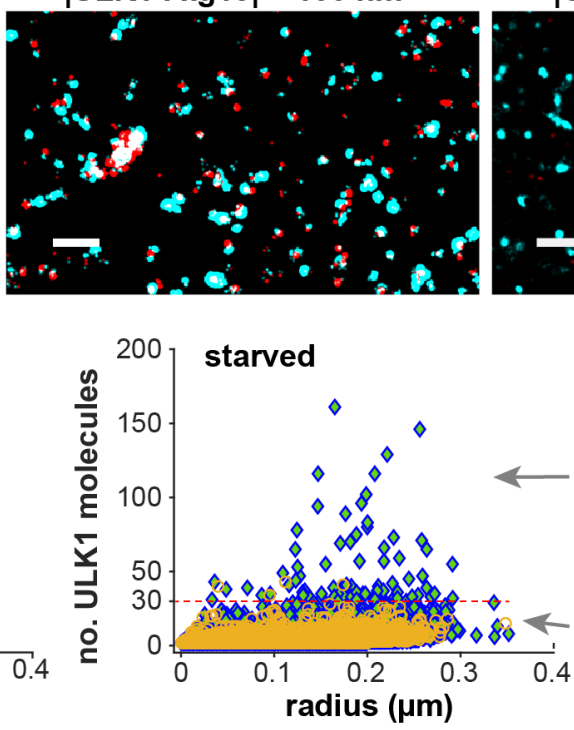

|ULK1-Atg13| > $100 \mathrm{~nm}$
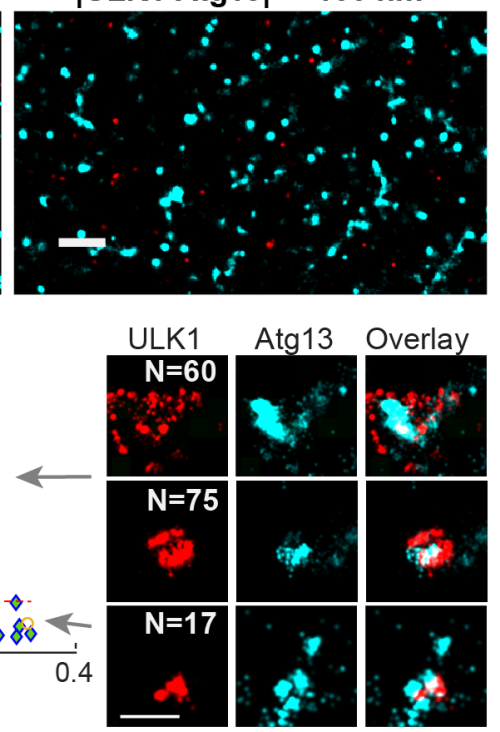
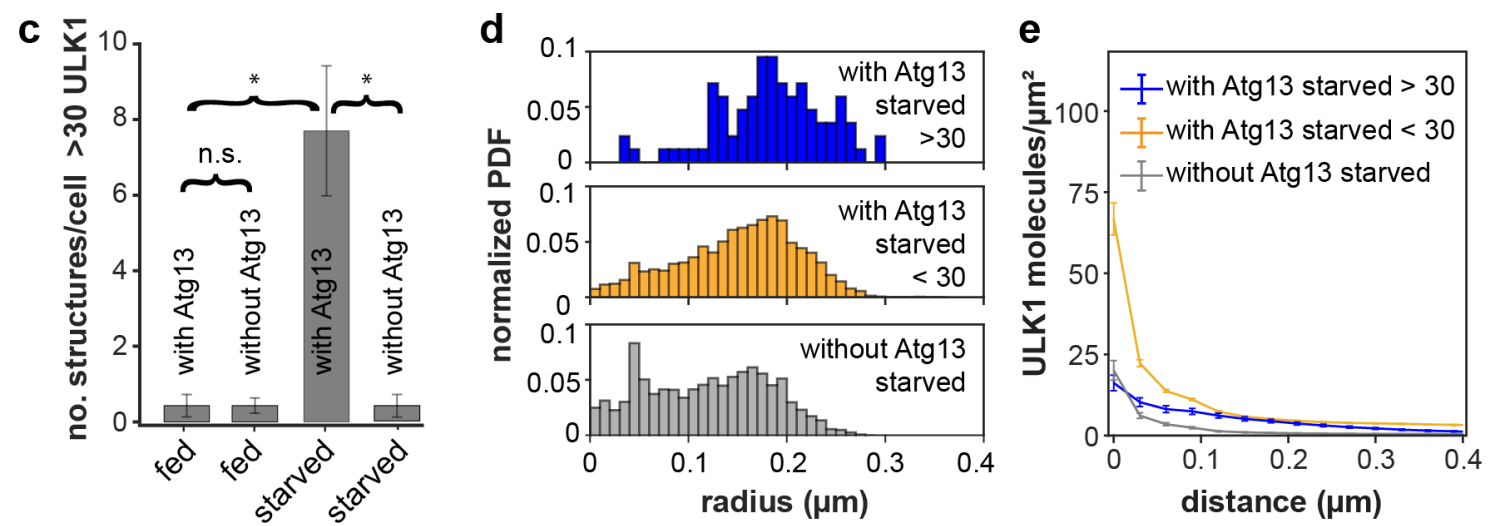

Fig.5. Cross-correlation analysis of two-color PALM data identifies starvation-induced ULK1 structures containing Atg13. a Left: Two-color PALM image of all mEos2-ULK1 (red) and Atg13-HaloTag JF646 (cyan) in starved cells. Middle: ULK1 molecules and clusters within $100 \mathrm{~nm}$ separation of Atg13 form highly dense and larger arc- or spherical structures. Right: ULK1 separated by more than $100 \mathrm{~nm}$ from Atg13 only forms small clusters. Scale bar: $1 \mu \mathrm{m}$. b Quantification of the number of molecules and radius of ULK1 associated structures. ULK1 structures within a $100 \mathrm{~nm}$ distance of Atg13 (with Atg13, blue) were compared to ULK1 structures not in proximity to Atg13 (without Atg13, orange). Left: ULK1 structures exhibited similar distributions in fed cells regardless of Atg13-bound states. Middle: small, dense ULK1 clusters as well as larger ULK1 structures with up to 161 ULK1 molecules form in starvation only when ULK1 structures are bound with Atg13. The dashed line at 30 molecules indicates a threshold used for further analysis. Right: the inset images exemplify large Atg13-containing structures as well as a small dense cluster with less ULK1 molecules under starvation. Scale bar: $500 \mathrm{~nm}$. c Number of structures with more than 30 ULK1 molecules per cell in different conditions. In fed cells, there was no significant difference between the number of structures with or without Atg13 per cell, whereas a significant difference was shown with starved cells (ANOVA, $p=0.05$ ). Error bars are SEM. d Probability density function (PDF) of the radii of structures with more than 30 ULK1 molecules with Atg13, with more than 30 ULK1 molecules without Atg13 (middle), and all other ULK1 molecules without Atg13. e Pair-correlation quantifies the density of ULK1 
molecules as a function of their distance on the three different types of ULK1 structures. Data was recorded from seven fed and ten starved cells.

a

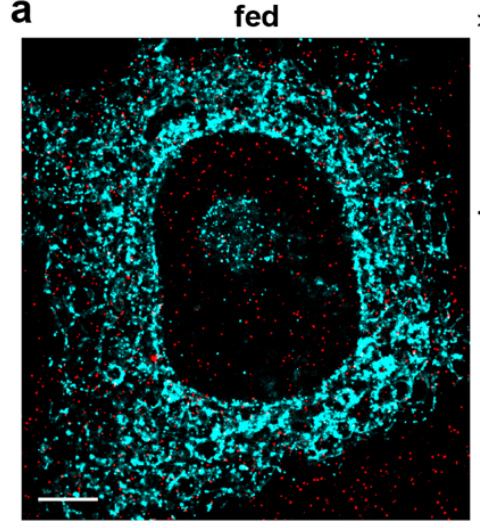

$>100 \mathrm{~nm}$ to $\mathrm{ER} ;<30$ ULK1

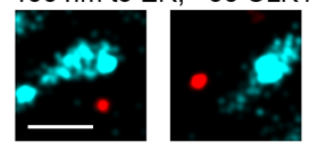

$<100 \mathrm{~nm}$ to ER; $<30$ ULK1
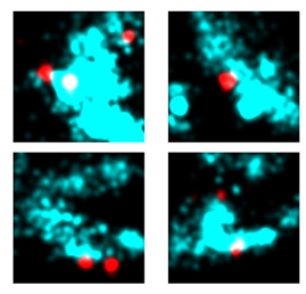

b
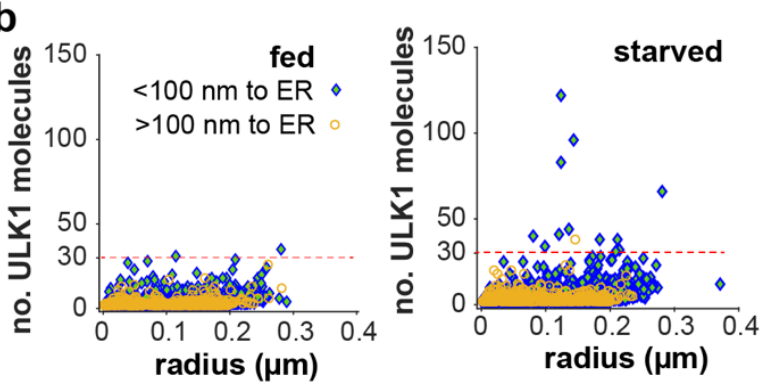

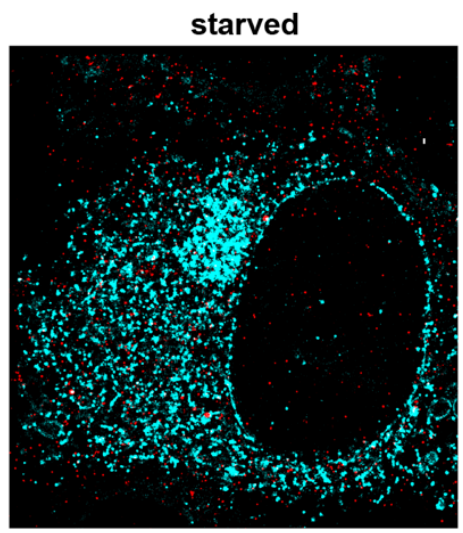

$>100 \mathrm{~nm}$ to ER; $<30$ ULK1

C

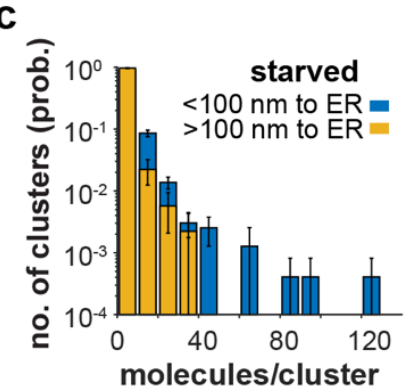

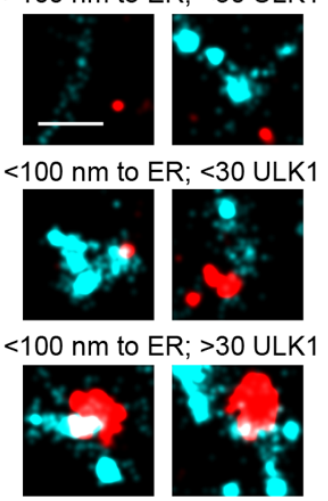

d

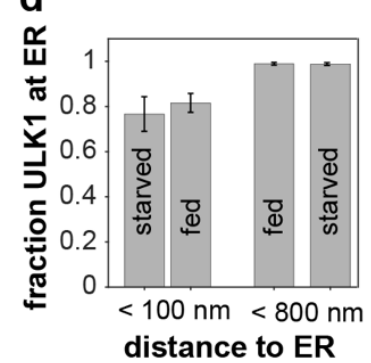

Fig. 6. Starvation induced ULK1 structures are in proximity to the ER. a Two color PALM images of mEos2-ULK1 (red) and the ER marker HaloTag-Sec61 $\beta$ (cyan) in fed and starved cells. Upper scale bar, $5 \mu \mathrm{m}$. Zoom images of ULK1 and the ER show the presence of large ULK1 clusters only on or in close proximity to the ER in fed cells. Scale bar: $1 \mu \mathrm{m}$. In starved cells larger ULK1 structures with more than 30 ULK1 molecules are in contact or in proximity to the ER (lower right). b Quantification of the number of ULK1 molecules and the approximated radius of structures that are or are not in close proximity of the ER in fed and starved cells. Structures with a high number of ULK1 molecules that were earlier identified to contain Atg13 are only found in close proximity to the ER in starved cells. c Normalized histogram of the number of ULK1 molecules in structures of starved cells. Structures in proximity to the ER exhibit the highest number of ULK1 molecules. d Fraction of ULK1 molecules at a given distance from the ER in fed and starved cells. In both fed and starved cells, about $80 \%$ of all ULK1 molecules were within a distance of $100 \mathrm{~nm}$ from an ER localization whereas over 95\% of ULK1 molecules were within a distance of $800 \mathrm{~nm}$. Errors represent SEM from four fed and five starved cells. 